\title{
Rare Disease Drug Policy and Inheritance and Innovation of Traditional Chinese Medicine
}

\author{
Baxian $\mathrm{Yi}^{1,2}$, Guangping Wang ${ }^{3} \&$ Xiaoming $\mathrm{Wu}^{1}$ \\ ${ }^{1}$ China Pharmaceutical University, Nanjing, China \\ ${ }^{2}$ China State Institute of Pharmaceutical Industry, Shanghai, China \\ ${ }^{3}$ Shanghai Institute for Food and Drug Safety, Shanghai, China \\ Correspondence: Xiaoming $\mathrm{Wu}$, China Pharmaceutical University, Nanjing 210009, China. E-mail: \\ xmwu@cpu.edu.cn
}

Received: November 17, 2013

doi:10.5539/ass.v10n3p181
Accepted: December 11, 2013 Online Published: January 27, 2014

URL: http://dx.doi.org/10.5539/ass.v10n3p181

\begin{abstract}
Traditional Chinese medicine is an important component of the health care system in Chinese medical treatment and public health. Rare disease drugs in difficult miscellaneous diseases in traditional Chinese medicine have similar characteristics with the rare disease drugs (orphan drugs) in European and American countries, which both are characterized by individualized medical treatment. The two are different in the development thought. Treatment of difficult miscellaneous diseases in the traditional Chinese medicine is based on the theoretical system of drug incompatibility, whereas orphan drugs in European and American countries lay particular stress on the gene therapy in molecular biology. Adoption of positive incentive policy and excavating folk remedy in the traditional Chinese medicine will promote the market position of the traditional Chinese medicine in the field of international rare disease drugs.
\end{abstract}

Keywords: traditional Chinese medicine, rare disease, orphan drug, difficult miscellaneous disease

At present, quite a lot of countries and regions regard rare disease drugs as an important component of the system of medical care insurance to ensure that the patients and their family share the rights of public health and health care. Since rare diseases belong to minority diseases, and their target market is small and the clinical experiment costs are high, almost all large-size transnational medical corporations, such as, Sanofi Aventis, GlaxoSmithKline, Pfizer and so on, focus more on development of rare disease drugs ever than before. As a supplement therapy of modern medicine, the traditional Chinese medicine has an application history for several thousand years and is characterized by individualized medical treatment. Especially, treatment of difficult miscellaneous diseases in the traditional Chinese medicine has common characteristics with treatment of orphan diseases in Europe and America. Research and development of the traditional Chinese medicine by referring to the guidance policies in foreign orphan drugs is inheritance and innovation of the traditional Chinese medicine.

1. The Traditional Chinese Medicine Is an Important Component of the System of China Medical Treatment

\subsection{The Traditional Chinese Medicine Is One of the Systems of China Basic Medical Treatment and Public Health}

Modern medicine is founded on the basis of the theory and technology of modern natural science and enters, step by step, the level of molecular mechanism. Although large scale of infectious diseases have been brought successfully under control and new diagnosis and treatment techniques and drugs emerge in endlessly, chronic diseases, iatrogenic and drug induced diseases continuously present a rising tendency. Thus, the traditional diagnosis and treatment technique and precaution means of "returning to the nature" arouse gradually more and more attention from all over the world. In the world traditional Chinese medicine, the traditional Chinese medicine, without doubt, is the best one developed and has assumed quite a large proportion of heavy burden in medical treatment and public health.

The traditional Chinese medicine was termed as Chinese materia medica and crude drug before the Qing Dynasty, which referred to drugs applied under the guidance of the basic theory of the traditional Chinese medicine. "Since Chinese herbal medicine in the Chinese medicine occupies a large proportion, books are called 
"Herbary" which record the Chinese medicine (Selected from traditional Chinese medicine [OL] Encyclopedia of China (chemical industry), (1905-06-14). Reference books online - National Library of China National Digital Library of China). In November 2008, "Beijing Declaration" of the WHO Congress on Traditional Medicine put forward the definition of "traditional medicine", which is "a sum of the knowledge, skills and practice based on intrinsic, explainable or unexplainable theory, belief and experience in different cultures and applied in protecting health and preventing, diagnosing, improving or curing somatopsychic diseases". US FDA mentioned in "Whole Medical Systems: An Overview" in 2004 that the system of the traditional Chinese medicine included Acupuncture, Massage and Chinese Materia Medica. The Chinese Materia Medica mentioned by FDA has similar concepts with the Chinese herbal medicine, traditional Chinese medicinal materials or Traditional Chinese Medicine decoction pieces in China.

For the time being, the traditional Chinese medicine has spread over more than 160 countries and regions. China has set up inter-governmental exchange and cooperation mechanism in terms of Chinese medicine or traditional medicine with approximately more than half of all countries and regions in the world. The $62^{\text {nd }}$ "World Health Assembly" passed "The Traditional Medicine Resolution" to urge its member countries to bring the traditional medicine into the national health service system. "The $12^{\text {th }}$ Five-Year Planning Outline of National Economy and Social Development" lists "support of development of Chinese medicine" as one of the six key tasks in "improvement of the basic medical care and public health system", which is a major component of the basic medical treatment and public health system in China. In the meantime, "National Essential Drugs List" (2012 version) contains 203 Chinese patent medicine varieties.

\subsection{Development of the Traditional Chinese Medicine Is a Basic National Policy of Chinese Government}

Chinese medicine industry is one of the important components in the public health and health care in Chinese medicine and development of the traditional Chinese medicine has, all along, been a significant national policy of the Chinese government. For example, in December 1982, the Chinese "Constitution" listed "development modern medicine and the traditional Chinese medicine". In 2011, "The $12^{\text {th }}$ Five-Year Planning Outline of National Economy and Social Development" regarded "support of development of Chinese medicine" as an important component of the medical treatment and public health system in China. "The $12^{\text {th }}$ Five-Year Planning for Development of Chinese Medicine" proposes the development goals that by the end of the " $12^{\text {th }}$ Five-Year", "Chinese medical treatment service system will be basically complete and the prevention and healthcare service network of Chinese medicine will be initially established and will play a more and more important role in construction of the basic medical care system".

International exchange and cooperation of traditional Chinese medicine is an effective approach for Chinese government to develop and improve traditional Chinese medicine. The traditional Chinese medicine has gone through two high tides in its stepping towards the world. The first tide is between AD 6-8 centuries when the Chinese medicine spread all eastern countries, including the Chinese prescription in Japan at present, the Korean medical science and eastern medical science in South Korea and eastern medical science in Vietnam, etc. The second tide is the period ever since the $70 \mathrm{~s}$ in the $20^{\text {th }}$ Century when the Chinese medicine gradually walks towards internationalization and acupuncture takes the lead in stepping towards the world. In 1972 when the departed US president Nixon paid a visit to China, acupuncture anesthesia ever caused a fever of acupuncture in US and stirred up study of China, US and WHO on acupuncture therapy which rapidly spread to more than 140 countries and regions in the world. In 2006, the Ministry of Science and Technology promulgated "International Scientific Cooperation Planning Outline of Chinese Medicine (2006-2020)" which proposed the guidance principle of "taking full advantage of global scientific resources to resolve key scientific issues in modernization of the Chinese medicine" and "regarding originality as the root and internationalization as the motive power". The aim was to further expand the opportunities of the traditional Chinese medicine in international exchange and cooperation. "Long and Medium Term Development Planning Outline for Standardization of Chinese Medicine (2011-2020)" proposed "finishing revision of 300 standardization systems for Chinese medicine by taking into consideration of the development of Chinese medicine, which almost covers the fields of Chinese medicine treatment, precaution and healthcare, education, scientific research and Chinese medicine". This is aimed to get mastery of the situation of international standardization development of Chinese medicine and stimulate rapid development of domestic Chinese medicine.

\section{Related Concepts in the Traditional Chinese Medicine for Treatment of Difficult Miscellaneous Diseases and Rare Disease Drugs}

\subsection{The Concept of Difficult Miscellaneous Diseases in the Traditional Chinese Medicine}

The traditional Chinese medicine is a precious treasury and treatment of difficult miscellaneous diseases in the 
Chinese medicine is a major subject in study on the traditional Chinese medicine science. Difficult miscellaneous disease has a lot of features and a variety of content. When the traditional Chinese medicine plays a more and more important role in the Chinese medical care insurance system, activities of research on difficult miscellaneous diseases, in recent years, have been active. This helps to facilitate development of scientific research of the traditional Chinese medicine in diagnosis and treatment of difficult miscellaneous diseases and enhance the medical care level of the vast majority of traditional Chinese medicine practitioners in diagnosis and treatment of difficult miscellaneous diseases.

Research on difficult miscellaneous diseases was earlier recorded in "The Inner Canon of Huangdi" in AD 475 during the Spring and Autumn and Warring States period (Lv, 1992, March). "Interrogation - Discussion on Strange Diseases" decided the symptoms of complicated condition of a disease, extensive scope of a disease, suspected symptoms, intermingled deficiency and excess and difficult to be cured as a difficult miscellaneous disease". In the theoretical system of the traditional Chinese medicine, diagnosis and treatment of difficult miscellaneous diseases is based on certain subjective content. For example, in AD 219 during the Spring and Autumn and Warring States period, the traditional medical scientist Zhang Zhonging made a detailed observation on typhoid fever and compiled "Treatise on Febrile and Miscellaneous Diseases". In AD 610, Chao Yuanfang et al. worked out "General Treatise on the Cause and Symptoms of Diseases" and pioneered discussion of the traditional Chinese medicine on all kinds of difficult miscellaneous diseases in the clinical centers of internal medicine, department of surgery, gynecology, department of pediatrics and ophthalmology and otorhinolaryngology. Difficult miscellaneous diseases are distinguished from critical diseases, strange diseases and modern diseases that are difficult to be cured. Difficult miscellaneous disease is a general concept and stubborn diseases are included in the scope of difficult miscellaneous diseases. Difficult miscellaneous diseases include "suspicious diseases", "difficult diseases" and "miscellaneous diseases". "Suspicious diseases" refer to the diseases that are in doubt (Xia, 1994, February), that is, diseases with ambiguous pathogeny, pathology and diagonosis and inefficient pharmacy; "difficult diseases" refer to those diseases which although are clear in terms of pathogeny, pathology and diagnosis, have no corresponding cure method or drug to use, as a result of which the effect is not perfect; by contrast, "miscellaneous diseases" include a lot of symptomatic diseases, either one kind of symptom, or a group of symptoms, which are deficient of explicit internal correlation, and which, thus, are neither able to be diagnosed in a systematic way nor able to be cured in a corresponding way. In the traditional Chinese medical theory which includes such treatment thoughts as specimen treatment, stasis treatment, sputum treatment, spleen and stomach treatment as well as kidney treatment, activating blood and dissolving stasis is one of the basic theories in curing difficult miscellaneous diseases. This was recorded in "Huangdi's Internal Classics", namely, the so-called "blood-excess syndrome should be treated by removing therapy".

\subsection{Similarities and Differences between Difficult Miscellaneous Diseases and Rare Diseases}

People's knowledge in diseases starts from exceptional and minority cases to general cases, that is, from rare disease $\rightarrow$ infrequent disease $\rightarrow$ common disease $\rightarrow$ frequently encountered disease. There are quite a large part of difficult miscellaneous diseases in the clinic which, to a certain extent, fall within the scope of rare diseases. The concepts of rare disease and orphan drug (rare disease drug) are "imported goods". The international society has no unified definition or definition standard on rare diseases. WHO defines rare disease as a single disease with a prevalence rate between $0.65 \%-1 \%$. Since rare diseases have a minority number of patients, small market demand and high research and development costs, few pharmaceutical enterprises focus on the research and development of the drugs for treatment of these diseases. Therefore, these drugs are vividly called "orphan drugs". The concept of the traditional Chinese medicine that cures difficult miscellaneous diseases is different from the concept of rare disease. The definition of rare disease makes classification based on the proportion of the treatment objects, whereas the difficult miscellaneous diseases make analysis from the theory and means of treatment, that is, diseases that can't be cured with the conventional treatment theory and means.

Difficult miscellaneous disease in the traditional Chinese medicine is a title on the diseases are difficult to treat with the traditional Chinese medical theory of simplicity holism, whereas rare disease is due to the proportion of the number of patients in the entire population based on the molecular organism of reductionism. For instance, US "Orphan Drug Act" defines rare disease as diseases with a prevalence rate lower than $0.75 \%$, the European Union "Regulation (EC) No 141/2000 on Orphan Medicinal Products" defines rare disease as diseases with a prevalence rate lower than $0.5 \%$ in the 27 countries of EU, Japan "Orphan Drug Regulation" defines rare disease as diseases with a prevalence rate lower than $0.4 \%$ and Australia "Orphan Drug Program" defines rare disease as diseases with a prevalence rate lower than $0.1 \%$.

In brief, although difficult miscellaneous diseases and rare diseases are different in terms of concept and scope, 
they have the common features of ambiguous disease pathogeny, unclear pathogenesis, lack of treatment means or ineffective drugs. Both of the two have single treatment projects and are characterized by individualized medical care. Difficult miscellaneous diseases in the traditional Chinese medicine use natural drugs and are lacking in diagnosis and treatment theory and effective methods. By contrast, although rare diseases have clear pathogeny mechanism, the enterprises are deficient of drug research and development motive as a result of low prevalence rate and low market efficiency.

\section{Research and Development of Rare Disease Drugs in Traditional Chinese Medicine}

\subsection{Protection of Patent System to the Traditional Chinese Medicine}

International Patent Classification (IPC) is a unique instrument that is compiled for classification of patent technology based on "International Patent classification Agreement/Strasbourg agreement" signed in 1971. There is no classification number of Chinese medicine theory in IPC. The original classification number of the material basis for efficacy in application for invention patent of the traditional Chinese medicine is determined by the origin of the raw materials of Chinese medicine. This sort of patent classification method is difficult to carry out effective protection to development of the traditional Chinese medicine.

The diagnosis and treatment method of the traditional drugs for difficult miscellaneous diseases or intellectual property protection of compatibility of Chinese herbal medicine is different from chemical drugs. The molecular structure of chemical drugs or the synthetic method is easy to identify and is relatively easy to get protection in pursuant to the patent law. By contrast, the traditional Chinese medicine depends more on the compatibility idea of the traditional Chinese medicine and on more diagnosis and treatment practice experiences and it is difficult to carry out rational protection to the compatibility idea and treatment experience in pursuant to the patent law. Therefore, the development thought of rare disease drugs in the traditional Chinese medicine is different from the development thought of orphan drugs in Europe and America. Drugs for treatment of difficult miscellaneous diseases in the traditional Chinese medicine are based on the theoretical system of compatibility, while orphan drugs in Europe and America lay particular stress on the gene treatment of molecular biology. On the condition of effectively protecting the intellectual property rights of the traditional Chinese medicine, bringing rare disease drugs in the traditional Chinese medicine into the category of medical care insurance drugs will more reflect the health service tenet of the national medical care insurance system.

\subsection{Adoption of Incentive Policy to Promote Development of the Traditional Chinese Medicine}

Since orphan drugs in Europe and America have a small target market and occupy a monopoly position, the government makes independent pricing to encourage innovation behavior of enterprises, and the price is generally high. And the government takes such administrative measures as innovation research and development incentive policy, production preferential tax policy and market guarantee policy related with orphan drugs to ensure accessibility of orphan drugs and guarantee maximization of social public health and welfare (Zeng Zhi, Yang Rui \& Linghu Changli, 2009). In contrast, rare disease drugs in the traditional Chinese medicine come from natural flora and fauna and the production costs of drugs are easy to take under control, which seems especially important for the governmental incentive policy at a research and development stage. It is quite necessary for the government to take sentinel procurement policy, government capital investment, preferential tax guiding policy to improve enthusiasm of research, development and production of drugs for difficult miscellaneous diseases in the traditional Chinese medicine.

How rare disease drugs in the traditional Chinese medicine are priced will affect efficiency and effect of development of the traditional Chinese medicine. Bringing drugs for difficult miscellaneous diseases and rare diseases in the traditional Chinese medicine into the medical care insurance system of the country or a region, on one hand, enhances accessibility of rare disease drugs in the traditional Chinese medicine, and, on the other hand, stimulates the innovative motive of traditional Chinese medicine enterprises. The government realizes national and regional medical care insurance coverage ratio to enhance the overall level of the national medical care insurance by means of constructing a price negotiation mechanism of rare disease drugs of the traditional Chinese medicine, which includes either government capital investment at an early stage or sentinel procurement at a later stage.

\subsection{Positively Excavating Chinese Domestic Folk Remedy and Folk Prescription}

The chemical synthesis that is totally based on cell-animal-clinic and medical development mode based on gene treatment have too high costs and the efficiency is low. The development thought of orphan drugs in Europe and America are more based on the molecular biological treatment with a thinking mode of reductionism, namely, gene treatment. As rare diseases belong to minority diseases and their target market is small and the clinical 
experiment costs are high, research and production enterprises of rare disease drugs are lacking in enthusiasm. Besides, the government gives a market monopoly period, which leads to expensive price of rare disease drugs. In the situation when the medical care insurance system is not yet able to cover the orphan drugs, the orphan drugs in Europe and America based on molecular mechanism are unable to satisfy the health demand of the vast majority of patients. Treatment of difficult miscellaneous adopts Chinese medicine, acupuncture or other substitution therapy. The traditional Chinese medicine usually comes from natural drugs and the production and treatment costs are far lower than investment of rare diseases at an early stage. Folk remedy (also called folk prescription, traditional cure or secret recipe) is an effective prescription which is popular in the folk and which is used to prevent and treat diseases. It is a crystallization of folk medical experiment experience and has the features of simple, convenient, cheap and experienced (Xu et al., 2009). It comes directly from the practical experience in treating diseases in the folk. Ge Hong in the Eastern Jin Dynasty collected simple, convenient, cheap and experienced home remedy and proved recipe in the folk and compiled "Prescription of Curing the Dead from Posterior Elbow" and "Taiping Prescription of Holy Benevolence" which collected 16834 prescriptions as well as "General Collection for Holy Relief" which collected more than 2000 prescriptions (Zhang \& Lu, 2001). "Prescriptions for Universal Relief" in the Ming Dynasty is the largest medical formulary in the ancient China (Chen, 2000), in which a lot of prescriptions originated from the folk. Therefore, exploring proved recipe in treatment of difficult miscellaneous diseases in the traditional Chinese medicine will effectively promote the important role of the traditional Chinese medicine in the international medicine market of rare diseases treatment.

\section{Concluding Remarks}

The traditional Chinese medicine that has an application history of several thousand years is an important component of the medical treatment and health care system in China. Similar to the rare disease and orphan drugs in countries and regions in Europe, America and Japan, rare disease drugs in treatment of difficult miscellaneous diseases in the traditional Chinese medicine are different in terms of drug development and production and focus more on excavation of the theoretical system of drug incompatibility. The traditional Chinese medicine has the clinical application characteristics of "simple, convenient and cheap" and its development and utilization of rare disease drugs in treatment of difficult miscellaneous diseases will enhance the position of the traditional Chinese medicine in the medical market of international rare diseases and orphan drugs.

\section{References}

Chen, M. Z. (2000). China Medical History. Beijing: People's Medical Publishing House.

Lv, C. Q. (1992, March). Diagnosis and Treatment of Difficult and Miscellaneous Diseases in Chinese Medicine (1st ed.). Beijing: Zhongyuan Farmers' Press.

Xia, G. H. (1994, February). Clinical Experience of Difficult and Miscellaneous Diseases (1st ed.). Beijing: Zhongyuan Farmers' Press.

Xu et al. (2009). Study on Collection of Folk Prescription and Proved Recipe of Chinese Medicine in Lingnan. Zhong Yao Cai, 32(1), 162-165.

Zeng, Z., Yang, R., \& Linghu, C. L. (2009). Discussion and Suggestions for the Planned Construction of the Rare Disease Drug Policy and System in China. China Pharmacy, (10), 737-740.

Zhang, J. W., \& Lu, Z. L. (2001). Reviews on prescription formula origination and development. Chinese Journal of Information on Traditional Chinese Medicine, 8(3), 6-81.

\section{Copyrights}

Copyright for this article is retained by the author(s), with first publication rights granted to the journal.

This is an open-access article distributed under the terms and conditions of the Creative Commons Attribution license (http://creativecommons.org/licenses/by/3.0/). 